\title{
ARITHMETIC FUNCTIONS ASSOCIATED WITH THE INFINITARY DIVISORS OF AN INTEGER
}

GRAEME L. COHEN and PETER hagis, JR.

School of Mathematical Sciences University of Technology, Sydney Broadway, NSW 2007, Australia
Department of Mathematics Temple University

Philadelphia, PA 19122

(Received November 21, 1991)

ABSTRACT. The infinitary divisors of a natural number $n$ are the products of its divisors of the form $p^{y_{\alpha} 2^{\alpha}}$, where $p^{y}$ is a prime-power component of $n$ and $\sum_{\alpha} y_{\alpha} 2^{\alpha}$ (where $y_{\alpha}=0$ or 1 ) is the binary representation of $y$. In this paper, we investigate the infinitary analogues of such familiar number theoretic functions as the divisor sum function, Euler's phi function and the Möbius function.

Key Words AND Phrases. Infinitary divisors, arithmetic functions, infinitary convolutions, asymptotic formulae.

1980 Mathematics Subject Classification. 11A25, 11 N37.

\section{Some Preliminaries}

Let $I=\left\{p^{2^{\alpha}}: p\right.$ is a prime and $\alpha$ is a nonnegative integer $\}$. If $n$ is a natural number greater than 1 then it follows easily from the fundamental theorem of arithmetic and the fact that the binary representation of a natural number is unique that $n$ can be written in exactly one way (except for the order of the factors) as a product of distinct elements from $I$. We shall call each element of $I$ in this product an $I$-component of $n$, and we shall say that $d$ is an $I$-divisor of $n$ if every $I$-component of $d$ is also an $I$-component of $n$. From the discussion in the first four sections of Cohen [1], it follows that the set of $I$-divisors of $n$ is equal to the set of infinitary divisors of $n$. (See Section 2 in Hagis and Cohen [2] for a concise definition of infinitary divisors.) If $d$ is an infinitary divisor (or $I$-divisor) of $n$, we write $\left.d\right|_{\infty} n$. For every natural number $n,\left.1\right|_{\infty} n$.

Now suppose that $n=P_{1} P_{2} \ldots P_{t}$, where $P_{1}<P_{2}<\cdots<P_{t}$ are the $I$-components of $n$. We shall denote $t$ by $J(n)$, so that $J(n)$ is the number of $I$-components of $n$ (with $J(1)=0$ ).

DEFINITION 1. The infinitary Möbius function, $\mu_{\infty}$, is given by

$$
\mu_{\infty}(n)=(-1)^{J(n)}
$$

and the infinitary phi function, $\phi_{\infty}$, is given by

$$
\phi_{\infty}(1)=1, \quad \phi_{\infty}(n)=\prod_{j=1}^{J(n)}\left(P_{j}-1\right)=n \prod_{j=1}^{J(n)}\left(1-\frac{1}{P_{j}}\right) \quad \text { if } n>1 .
$$

According to Theorem 13 in [1], if $\tau_{\infty}(n)$ and $\sigma_{\infty}(n)$ denote the number and sum, respectively, of the infinitary divisors of $n$, then:

$$
\begin{aligned}
\tau_{\infty}(n) & =2^{J(n)}, \\
\sigma_{\infty}(n) & =\prod_{j=1}^{J(n)}\left(P_{j}+1\right) .
\end{aligned}
$$


REMARK 1. We have $\sigma_{\infty}(n) \phi_{\infty}(n)=\phi_{\infty}\left(n^{2}\right)$.

DEFINITION 2. If $d$ is the greatest common infinitary divisor of the natural numbers $m$ and $n$, we shall write $(m, n)_{\infty}=d$. If $(m, n)_{\infty}=1$, we shall say that $m$ and $n$ are $I$-prime.

REMARK 2. For every natural number $m,(m, 1)_{\infty}=1$.

REMARK 3. If $(m, n)=1$, then $(m, n)_{\infty}=1$.

REMARK 4. If $\left.d\right|_{\infty} n$, then $d$ and $n / d$ are $I$-prime.

DEFINITION 3. The arithmetic function $f$ (where $f$ is not identically zero) is said to be $I$-multiplicative if $(m, n)_{\infty}=1$ implies that $f(m n)=f(m) f(n)$.

REMARK 5. If $f$ is $I$-multiplicative, then $f$ is also multiplicative.

The following result is obvious.

THEOREM 1. Suppose that $f$ and $g$ are $I$-multiplicative functions. Then $f(1)=1$, and $f g$ and $|f|$ are $I$-multiplicative functions. If $g(n) \neq 0$ for every $n$, then $f / g$ is $I$-multiplicative.

From (1.1), (1.2), (1.3), (1.4), we have:

THEOREM 2. Each of the functions $\mu_{\infty}, \phi_{\infty}, \tau_{\infty}, \sigma_{\infty}$ is I-multiplicative.

THEOREM 3. Suppose that $f$ is an I-multiplicative function. If $F(n)=\sum_{\left.d\right|_{\infty} n} f(d)$ (where $d$ runs over all of the infinitary divisors of $n$ ), then $F$ is also an $I$-multiplicative function.

Proof. Suppose that $m$ and $n$ are $I$-prime. If $m=1$ or $n=1$ then (since $f(1)=1$ ) $F(m)=1$ or $F(n)=1$, and it follows that $F(m n)=F(m) F(n)$. Otherwise, since the infinitary divisors of a natural number are 1 and the set of all products of its $I$-components taken one at a time, two at a time, etc., we see that $d$ is an infinitary divisor of $m n$ if and only if $d=d_{1} d_{2}$ where $\left.d_{1}\right|_{\infty} m$ and $\left.d_{2}\right|_{\infty} n$. Of course, $d_{1}$ and $d_{2}$ are $I$-prime. Therefore,

$$
\begin{aligned}
F(m n) & =\sum_{\left.d\right|_{\infty} m n} f(d)=\sum_{\left.d_{1}\right|_{\infty} m} \sum_{\left.d_{2}\right|_{\infty} n} f\left(d_{1} d_{2}\right) \\
& =\sum_{\left.d_{1}\right|_{\infty} m} \sum_{\left.d_{2}\right|_{\infty} n} f\left(d_{1}\right) f\left(d_{2}\right)=\sum_{\left.d_{1}\right|_{\infty} m} f\left(d_{1}\right) \sum_{\left.d_{2}\right|_{\infty} n} f\left(d_{2}\right)=F(m) F(n) .
\end{aligned}
$$

THEOREM 4. We have $\sum_{\left.d\right|_{\infty} n} \phi_{\infty}(d)=n$.

Proof. Let $F(n)=\sum_{\left.d\right|_{\infty} n} \phi_{\infty}(d)$. It is immediate that $F(1)=1$. Also, if $n=P \in I$ then

$$
F(n)=\sum_{\left.d\right|_{\infty} n} \phi_{\infty}(d)=\phi_{\infty}(1)+\phi_{\infty}(P)=1+(P-1)=n .
$$

Since $\phi_{\infty}$ is an $I$-multiplicative function, it follows from Theorem 3 that $F$ is $I$-multiplicative. Therefore, if $n=P_{1} P_{2} \ldots P_{t}$, then $F(n)=\prod_{j=1}^{t} F\left(P_{j}\right)=\prod_{j=1}^{t} P_{j}=n$.

DEFINITION 4. The arithmetic function $i$, given by

$$
i(n)=\left[\frac{1}{n}\right]= \begin{cases}1, & \text { if } n=1, \\ 0, & \text { if } n>1,\end{cases}
$$

is called the identity function.

The proof of the following theorem is very similar to that of Theorem 4 and therefore is omitted.

THEOREM 5. We have $\sum_{\left.d\right|_{\infty} n} \mu_{\infty}(d)=i(n)$.

\section{The Infinitary Convolution}

In this section, we study the infinitary analogue of the familiar operation of Dirichlet convolution on arithmetic functions. Since the definitions and theorems parallel those to be found in Chapter Two of Apostol [3], we omit most of the proofs. 
DEFINITION 5. If $f$ and $g$ are arithmetic functions, their infinitary convolution, denoted $(f * g)_{\infty}$, is the arithmetic function given by

$$
(f * g)_{\infty}(n)=\sum_{\left.d\right|_{\infty} n} f(d) g\left(\frac{n}{d}\right) .
$$

THEOREM 6. For any arithmetic functions $f, g$, $h$, we have $(f * g)_{\infty}=(g * f)_{\infty}$ (the commutative law) and $\left((f * g)_{\infty} * h\right)_{\infty}=\left(f *(g * h)_{\infty}\right)_{\infty}$ (the associative law).

THEOREM 7. For every arithmetic function $f$, we have $(i * f)_{\infty}=(f * i)_{\infty}=f$.

THEOREM 8. If $f$ is an arithmetic function such that $f(1) \neq 0$, there is a unique arithmetic function $\left(f^{-1}\right)_{\infty}$, called the infinitary inverse of $f$, such that

$$
\left(f *\left(f^{-1}\right)_{\infty}\right)_{\infty}=\left(\left(f^{-1}\right)_{\infty} * f\right)_{\infty}=i .
$$

REMARK 6. From Theorems $6,7,8$, it follows that the set of all arithmetic functions $f$ with $f(1) \neq 0$ forms an abelian group with respect to the operation of infinitary convolution, the identity element being the function $i$.

DEFINITION 6. The unit function $u$ is the arithmetic function such that $u(n)=1$ for every natural number $n$.

From Theorem 5 and Definition 6 , we have $\left(\mu_{\infty} * u\right)_{\infty}=i$. Therefore, from Theorem 8 ,

$$
\left(\mu_{\infty}^{-1}\right)_{\infty}=u \text { and }\left(u^{-1}\right)_{\infty}=\mu_{\infty}
$$

THEOREM 9. (The Möbius inversion formula.) We have $F(n)=\sum_{d_{\left.\right|_{\infty} n}} f(d)$ if and only if $f(n)=\sum_{\left.d\right|_{\infty} n} F(n / d) \mu_{\infty}(d)$.

Proof. Suppose that $F=(f * u)_{\infty}$. Then $\left(F * \mu_{\infty}\right)_{\infty}=\left((f * u)_{\infty} * \mu_{\infty}\right)_{\infty}=$ $\left(f *\left(u * \mu_{\infty}\right)_{\infty}\right)_{\infty}=(f * i)_{\infty}=f$. Conversely, suppose that $f=\left(F * \mu_{\infty}\right)_{\infty}$. Then $(f * u)_{\infty}=\left(\left(F * \mu_{\infty}\right)_{\infty} * u\right)_{\infty}=\left(F *\left(\mu_{\infty} * u\right)_{\infty}\right)_{\infty}=(F * i)_{\infty}=F$.

COROllaRY 9.1. We have $\phi_{\infty}(n)=\sum_{\left.d\right|_{\infty} n} \mu_{\infty}(d)(n / d)=n \sum_{\left.d\right|_{\infty} n} \mu_{\infty}(d) / d$.

Proof. From Theorem 4, $n=\sum_{\left.d\right|_{\infty} n} \phi_{\infty}(d)$. Our result follows from Theorem 9.

THEOREM 10. If $f$ and $g$ are each I-multiplicative, then so is $(f * g)_{\infty}$.

THEOREM 11. If $g$ and $(f * g)_{\infty}$ are each $I$-multiplicative functions, then $f$ is also $I$ multiplicative.

CoROLlaRY 11.1. If $g$ is I-multiplicative, so is its infinitary inverse $\left(g^{-1}\right)_{\infty}$.

Proof. Both $g$ and $i=\left(g *\left(g^{-1}\right)_{\infty}\right)_{\infty}$ are $I$-multiplicative. Therefore, $\left(g^{-1}\right)_{\infty}$ is $I$ multiplicative.

REMARK 7. It follows from Theorems $6,7,8,10$ and Corollary 11.1 that the set of $I$ multiplicative functions is a subgroup of the group of arithmetic functions $f$ such that $f(1) \neq 0$.

THEOREM 12. If $f$ is I-multiplicative, then $(f * f)_{\infty}=f \tau_{\infty}$.

Proof. We have

$$
(f * f)_{\infty}(n)=\sum_{\left.d\right|_{\infty} n} f(d) f\left(\frac{n}{d}\right)=\sum_{\left.d\right|_{\infty} n} f(n)=f(n) \sum_{\left.d\right|_{\infty} n} 1=f(n) \tau_{\infty}(n) .
$$

TheOREM 13. If $f$ is I-multiplicative, then $\left(f^{-1}\right)_{\infty}=f \mu_{\infty}$.

Proof. Let $g(n)=f(n) \mu_{\infty}(n)$. Then 


$$
\begin{aligned}
(g * f)_{\infty}(n) & =\sum_{\left.d\right|_{\infty} n} \mu_{\infty}(d) f(d) f\left(\frac{n}{d}\right)=\sum_{\left.d\right|_{\infty} n} \mu_{\infty}(d) f(n) \\
& =f(n) \sum_{\left.d\right|_{\infty} n} \mu_{\infty}(d)=f(n) i(n)=i(n) .
\end{aligned}
$$

CoROLla RY 13.1. If $f$ is I-multiplicative, then $\left(f^{-1}\right)_{\infty}(n)=f(n)$ or $-f(n)$, according as $J(n)$ is even or odd.

\section{SOME SUMmatory FunCtions}

Throughout this section, we assume that if the natural number $n$ exceeds 1 , then $n=$ $P_{1} P_{2} \ldots P_{J}$, where $P_{1}<P_{2}<\cdots<P_{J}$ are the $I$-components of $n$. Also, $\epsilon$ will denote a real number satisfying $0<\epsilon \leq 1$.

DEFINITION 7. The arithmetic function $\kappa_{\infty}$ is defined by

$$
\kappa_{\infty}(1)=1, \quad \kappa_{\infty}(n)=\prod_{j=1}^{J} \frac{P_{j}}{P_{j}+1} \quad \text { if } n>1 .
$$

It is immediate that $\kappa_{\infty}$ is $I$-multiplicative, and that $\kappa_{\infty}(n) \leq 1$ for all $n$.

DEFINITION 8. If $x>0$ is a real number,

$$
\phi_{\infty}(x, n)=\sum_{\substack{m \leq x \\(m, n)_{\infty}=1}} 1 .
$$

REMARK 8. In general it is not true that $\phi_{\infty}(n, n)=\phi_{\infty}(n)$. See Corollary 14.1.

LEMMA 1. Let $P$ be any $I$-component of $n$, where $n>1$. Then

$$
\phi_{\infty}(x, n)=\phi_{\infty}\left(x, \frac{n}{P}\right)-\phi_{\infty}\left(\frac{x}{P}, n\right) .
$$

Proof. We have

$$
\begin{aligned}
\phi_{\infty}(x, n) & =\sum_{\substack{m \leq x \\
(m, n)_{\infty}=1}} 1=\sum_{\substack{m \leq x \\
(m, n / \bar{P})_{\infty}=1}} 1-\sum_{\substack{m \leq\left. x \\
P\right|_{\infty} m \\
(m / P, n)_{\infty}=1}} 1 \\
& =\phi_{\infty}\left(x, \frac{n}{P}\right)-\sum_{\substack{k \\
k P \leq x \\
(k, n)_{\infty}=1}} 1=\phi_{\infty}\left(x, \frac{n}{P}\right)-\sum_{\substack{k \leq x / P \\
(k, n)_{\infty}=1}} 1 \\
& =\phi_{\infty}\left(x, \frac{n}{P}\right)-\phi_{\infty}\left(\frac{x}{P}, n\right) .
\end{aligned}
$$

THEOREM 14. For any positive real number $x$ and any natural number $n$,

$$
\phi_{\infty}(x, n)=\kappa_{\infty}(n) x+O\left(n^{\epsilon} x^{\epsilon}\right)
$$

where the multiplicative constant implied by the big oh notation depends only on $\epsilon$.

Proof. The proof will include an estimation of the constant, $c_{\epsilon}$ say, implied by the big oh notation.

Let $Q$ be the smallest element of $I$ such that

$$
1-\frac{\log \left(Q^{\epsilon}-1\right)}{\log Q^{\epsilon}}<\epsilon
$$


and let

$$
\rho_{\epsilon}=\frac{Q^{\epsilon}}{Q^{\epsilon}-1}
$$

(For example, taking $\epsilon=0.8,0.5,0.3$ and 0.1 , we find that, respectively, $Q=3,11,107$ and 1876451. Since $2^{\epsilon}-1 \leq 1$, we see that $Q \geq 3$.)

Suppose first that $x<1$. Then $\phi_{\infty}(x, n)=0$, and

$$
\left|\phi_{\infty}(x, n)-\kappa_{\infty}(n) x\right|=\kappa_{\infty}(n) x \leq x \leq n^{\epsilon} x^{\epsilon}
$$

so (3.1) holds in this case, provided $c_{\epsilon} \geq 1$.

From now on, we assume that $x \geq 1$.

Suppose that $n=1$. Then $\phi_{\infty}(x, n)=[x]$, and

$$
\left|\phi_{\infty}(x, n)-\kappa_{\infty}(n) x\right|=x-[x]<1 \leq n^{\epsilon} x^{\epsilon},
$$

so (3.1) holds in this case as well, again provided $c_{\epsilon} \geq 1$.

We now assume further that $n>1$, and consider first those $n$ for which $Q \leq P_{1}$. We will show that, for any positive integer $k$ and all $x<P_{1}^{k \epsilon}$,

$$
\left|\phi_{\infty}(x, n)-\kappa_{\infty}(n) x\right|<2 \rho_{\epsilon}^{k} n^{\epsilon} .
$$

The proof is by induction on $k$.

When $k=1$, we have, since $x<P_{1}^{\epsilon}, 0<\kappa_{\infty}(n)<1$ and $\rho_{\epsilon}>1$,

$$
\left|\phi_{\infty}(x, n)-\kappa_{\infty}(n) x\right| \leq \phi_{\infty}(x, n)+\kappa_{\infty}(n) x<[x]+x \leq 2 x<2 P_{1}^{\epsilon} \leq 2 n^{\epsilon}<2 \rho_{\epsilon} n^{\epsilon},
$$

which is the required result in this case.

Now suppose the result is true when $k=l$, and consider those $x<P_{1}^{(l+1) \epsilon}$. For such $x$ and any $j, 1 \leq j \leq J$, we have $x / P_{j}<P_{1}^{l \epsilon}$.

By repeated application of Lemma 1 ,

$$
\begin{aligned}
\phi_{\infty}(x, n)= & \phi_{\infty}\left(x, \frac{n}{P_{1}}\right)-\phi_{\infty}\left(\frac{x}{P_{1}}, n\right) \\
= & \phi_{\infty}\left(x, \frac{n}{P_{1} P_{2}}\right)-\phi_{\infty}\left(\frac{x}{P_{2}}, \frac{n}{P_{1}}\right)-\phi_{\infty}\left(\frac{x}{P_{1}}, n\right) \\
= & \phi_{\infty}\left(x, \frac{n}{P_{1} P_{2} P_{3}}\right)-\phi_{\infty}\left(\frac{x}{P_{3}}, \frac{n}{P_{1} P_{2}}\right)-\phi_{\infty}\left(\frac{x}{P_{2}}, \frac{n}{P_{1}}\right)-\phi_{\infty}\left(\frac{x}{P_{1}}, n\right) \\
\vdots & \quad-\cdots-\phi_{\infty}\left(\frac{x}{P_{2}}, \frac{n}{P_{1}}\right)-\phi_{\infty}\left(\frac{x}{P_{1}}, n\right) \\
= & \phi_{\infty}(x, 1)-\phi_{\infty}\left(\frac{x}{P_{J}}, \frac{n}{P_{1} P_{2} \ldots P_{J-1}}\right)-\phi_{\infty}\left(\frac{x}{P_{J-1}}, \frac{n}{P_{1} P_{2} \ldots P_{J-2}}\right) \\
= & {[x]-\phi_{\infty}\left(\frac{x}{P_{J}}, P_{J}\right)-\phi_{\infty}\left(\frac{x}{P_{J-1}}, P_{J} P_{J-1}\right)-\cdots-\phi_{\infty}\left(\frac{x}{P_{2}}, \frac{n}{P_{1}}\right)-\phi_{\infty}\left(\frac{x}{P_{1}}, n\right) } \\
= & \quad x]-x+\left(1-\frac{\kappa_{\infty}\left(P_{J}\right)}{P_{J}}-\frac{\kappa_{\infty}\left(P_{J} P_{J-1}\right)}{P_{J-1}}-\frac{\kappa_{\infty}\left(P_{J} P_{J-1} P_{J-2}\right)}{P_{J-2}}-\cdots-\frac{\kappa_{\infty}(n)}{P_{1}}\right) \\
& \quad-\left(\phi_{\infty}\left(\frac{x}{P_{J}}, P_{J}\right)-\kappa_{\infty}\left(P_{J}\right) \frac{x}{P_{J}}\right) \\
& \quad-\left(\phi_{\infty}\left(\frac{x}{P_{J-1}}, P_{J} P_{J-1}\right)-\kappa_{\infty}\left(P_{J} P_{J-1}\right) \frac{x}{P_{J-1}}\right) \\
& -\cdots-\left(\phi_{\infty}\left(\frac{x}{P_{1}}, n\right)-\kappa_{\infty}(n) \frac{x}{P_{1}}\right) .
\end{aligned}
$$


Since

$$
\begin{aligned}
1- & \frac{\kappa_{\infty}\left(P_{J}\right)}{P_{J}}-\frac{\kappa_{\infty}\left(P_{J} P_{J-1}\right)}{P_{J-1}}-\frac{\kappa_{\infty}\left(P_{J} P_{J-1} P_{J-2}\right)}{P_{J-2}}-\cdots-\frac{\kappa_{\infty}(n)}{P_{1}} \\
= & 1-\frac{1}{P_{J}+1}-\frac{P_{J}}{P_{J}+1} \frac{1}{P_{J-1}+1} \\
& -\frac{P_{J}}{P_{J}+1} \frac{P_{J-1}}{P_{J-1}+1} \frac{1}{P_{J-2}+1}-\cdots-\frac{P_{J}}{P_{J}+1} \frac{P_{J-1}}{P_{J-1}+1} \cdots \frac{P_{2}}{P_{2}+1} \frac{1}{P_{1}+1} \\
= & \frac{P_{J}}{P_{J}+1}-\frac{P_{J}}{P_{J}+1} \frac{1}{P_{J-1}+1} \\
& -\frac{P_{J}}{P_{J}+1} \frac{P_{J-1}}{P_{J-1}+1} \frac{1}{P_{J-2}+1}-\cdots-\frac{P_{J}}{P_{J}+1} \frac{P_{J-1}}{P_{J-1}+1} \cdots \frac{P_{2}}{P_{2}+1} \frac{1}{P_{1}+1} \\
= & \frac{P_{J}}{P_{J}+1} \frac{P_{J-1}}{P_{J-1}+1} \\
& -\frac{P_{J}}{P_{J}+1} \frac{P_{J-1}}{P_{J-1}+1} \frac{1}{P_{J-2}+1}-\cdots-\frac{P_{J}}{P_{J}+1} \frac{P_{J-1}}{P_{J-1}+1} \cdots \frac{P_{2}}{P_{2}+1} \frac{1}{P_{1}+1} \\
\vdots & \\
= & \frac{P_{J}}{P_{J}+1} \frac{P_{J-1}}{P_{J-1}+1} \cdots \frac{P_{2}}{P_{2}+1} \frac{P_{1}}{P_{1}+1} \\
= & \kappa_{\infty}(n),
\end{aligned}
$$

we thus have, using the induction hypothesis,

$$
\begin{aligned}
\left|\phi_{\infty}(x, n)-\kappa_{\infty}(n) x\right| \leq x-[x] & +\left|\phi_{\infty}\left(\frac{x}{P_{J}}, P_{J}\right)-\kappa_{\infty}\left(P_{J}\right) \frac{x}{P_{J}}\right| \\
& \quad+\left|\phi_{\infty}\left(\frac{x}{P_{J-1}}, P_{J} P_{J-1}\right)-\kappa_{\infty}\left(P_{J} P_{J-1}\right) \frac{x}{P_{J-1}}\right| \\
& \quad+\cdots+\left|\phi_{\infty}\left(\frac{x}{P_{1}}, n\right)-\kappa_{\infty}(n) \frac{x}{P_{1}}\right| \\
& <1+2 \rho_{\epsilon}^{l}\left(P_{J}^{\epsilon}+\left(P_{J} P_{J-1}\right)^{\epsilon}+\left(P_{J} P_{J-1} P_{J-2}\right)^{\epsilon}+\cdots+n^{\epsilon}\right) \\
< & 2 \rho_{\epsilon}^{l}\left(1+P_{J}^{\epsilon}+\left(P_{J} P_{J-1}\right)^{\epsilon}+\left(P_{J} P_{J-1} P_{J-2}\right)^{\epsilon}+\cdots+n^{\epsilon}\right) \\
= & 2 \rho_{\epsilon}^{l} n^{\epsilon}\left(1+\frac{1}{P_{1}^{\epsilon}}+\frac{1}{\left(P_{1} P_{2}\right)^{\epsilon}}+\cdots+\frac{1}{\left(P_{1} P_{2} \ldots P_{J-1}\right)^{\epsilon}}+\frac{1}{n^{\epsilon}}\right) \\
& <2 \rho_{\epsilon}^{l} n^{\epsilon}\left(1+\frac{1}{Q^{\epsilon}}+\frac{1}{Q^{2 \epsilon}}+\frac{1}{Q^{3 \epsilon}}+\cdots\right) \\
& =2 \rho_{\epsilon}^{l+1} n^{\epsilon} .
\end{aligned}
$$

This completes the proof of (3.3).

Now, for given $x$ we let $k$ be the smallest integer such that $x<P_{1}^{k \epsilon}$. (Note that $k \geq 1$ since $x \geq 1$.) Then $x \geq P_{1}^{(k-1) \epsilon} \geq Q^{(k-1) \epsilon}$, so that $k-1 \leq \log x / \epsilon \log Q$. Then

$$
\rho_{\epsilon}^{k-1}=\left(\frac{Q^{\epsilon}}{Q^{\epsilon}-1}\right)^{k-1} \leq\left(\frac{Q^{\epsilon}}{Q^{\epsilon}-1}\right)^{\log x / \epsilon \log Q}=x^{\log \left(Q^{\epsilon} /\left(Q^{\epsilon}-1\right)\right) / \log Q^{\epsilon}}<x^{\epsilon},
$$

using (3.2). It therefore follows from (3.3) that (3.1) is true, provided $c_{\epsilon} \geq 2 \rho_{\epsilon}$, for all $n$ with smallest $I$-component at least $Q$. 
Consider next those $n$ with $P_{1}<Q \leq P_{2}$. By repeated application of Lemma 1 ,

$$
\begin{aligned}
\phi_{\infty}(x, n) & =\phi_{\infty}\left(x, \frac{n}{P_{1}}\right)-\phi_{\infty}\left(\frac{x}{P_{1}}, n\right) \\
& =\phi_{\infty}\left(x, \frac{n}{P_{1}}\right)-\phi_{\infty}\left(\frac{x}{P_{1}}, \frac{n}{P_{1}}\right)+\phi_{\infty}\left(\frac{x}{P_{1}^{2}}, n\right) \\
& \vdots \\
& =\phi_{\infty}\left(x, \frac{n}{P_{1}}\right)-\phi_{\infty}\left(\frac{x}{P_{1}}, \frac{n}{P_{1}}\right)+\phi_{\infty}\left(\frac{x}{P_{1}^{2}}, \frac{n}{P_{1}}\right)-\cdots \pm \phi_{\infty}\left(\frac{x}{P_{1}^{t}}, \frac{n}{P_{1}}\right) \mp \cdots
\end{aligned}
$$

This is a finite series, terminating after $t+1$ terms, where $x / P_{1}^{t+1}<1 \leq x / P_{1}^{t}$. Since the smallest $I$-component of $n / P_{1}$ is at least $Q$, the work above shows that

$$
\begin{aligned}
\phi_{\infty}(x, n)= & \kappa_{\infty}\left(\frac{n}{P_{1}}\right)\left(x-\frac{x}{P_{1}}+\frac{x}{P_{1}^{2}}-\cdots \pm \frac{x}{P_{1}^{t}}\right) \\
& +O\left(\left(\frac{n}{P_{1}}\right)^{\epsilon}\left(x^{\epsilon}+\left(\frac{x}{P_{1}}\right)^{\epsilon}+\left(\frac{x}{P_{1}^{2}}\right)^{\epsilon}+\cdots+\left(\frac{x}{P_{1}^{t}}\right)^{\epsilon}\right)\right) \\
= & \kappa_{\infty}\left(\frac{n}{P_{1}}\right)\left(1-\frac{1}{P_{1}}+\frac{1}{P_{1}^{2}}-\cdots\right) x+O\left(\frac{x}{P_{1}^{t+1}}\right) \\
& \quad+O\left(\left(\frac{n}{P_{1}}\right)^{\epsilon}\left(1+\frac{1}{P_{1}^{\epsilon}}+\frac{1}{P_{1}^{2 \epsilon}}+\cdots\right) x^{\epsilon}\right) \\
= & \frac{\kappa_{\infty}(n)}{\kappa_{\infty}\left(P_{1}\right)} \frac{P_{1}}{P_{1}+1} x+O(1)+O\left(n^{\epsilon} x^{\epsilon}\right) \\
= & \kappa_{\infty}(n) x+O\left(n^{\epsilon} x^{\epsilon}\right) .
\end{aligned}
$$

The extra multiplicative factor introduced in the error term is

$$
\frac{1}{P_{1}^{\epsilon}} \cdot \frac{P_{1}^{\epsilon}}{P_{1}^{\epsilon}-1} \leq \frac{1}{2^{\epsilon}-1}
$$

The $O(1)$ term in fact adds no more than 1 , which is less than $1 \cdot n^{\epsilon} x^{\epsilon}$, to the error term. Since $1+2 \rho_{\epsilon} /\left(2^{\epsilon}-1\right)<3 \rho_{\epsilon} /\left(2^{\epsilon}-1\right)$ (because $\left.\rho_{\epsilon}>1 \geq 2^{\epsilon}-1\right)$, we may take $c_{\epsilon} \geq 3 \rho_{\epsilon} /\left(2^{\epsilon}-1\right)$. Theorem 14 has thus been proved for those $n$ with at most one $I$-component less than $Q$.

If $Q>3$, then there exist $n$ for which $P_{2}<Q \leq P_{3}$. For such $n, n / P_{1}$ has at most one $I$-component less than $Q$, so precisely the same approach as in the preceding paragraph, using the result there, again shows Theorem 14 to be true, provided that we take $c_{\epsilon} \geq 4 \rho_{\epsilon} /\left(2^{\epsilon}-1\right)^{2}$.

By repeating this argument, we see that if $P_{s}<Q \leq P_{s+1}$, for some $s=1,2, \ldots, J-1$, then the theorem is true, with $c_{\epsilon} \geq(s+2) \rho_{\epsilon} /\left(2^{\epsilon}-1\right)^{s}$. (This statement also holds for $s=0$ if we define $P_{0}$ to be 1.) If $F(Q)$ denotes the number of elements of $I$ which are less than $Q$, and $P_{s}<Q \leq P_{s+1}$ where $s+1 \leq J$, then it is easy to see that $s \leq F(Q)$. It follows that if $x \geq 1$ and $Q \leq P_{J}$, then the theorem is true, provided we take $c_{\epsilon} \geq(F(Q)+2) \rho_{\epsilon} /\left(2^{\epsilon}-1\right)^{F(Q)}$.

We must consider finally those $n$ with $Q>P_{J}$. From Lemma 1,

$$
\phi_{\infty}(x, n Q)=\phi_{\infty}(x, n)-\phi_{\infty}\left(\frac{x}{Q}, n Q\right),
$$

and the result of the preceding paragraph implies that

$$
\phi_{\infty}(x, n)=\kappa_{\infty}(n Q) x+O\left(n^{\epsilon} Q^{\epsilon} x^{\epsilon}\right)+\kappa_{\infty}(n Q) \frac{x}{Q}+O\left(n^{\epsilon} x^{\epsilon}\right) .
$$

It now follows easily that

$$
\phi_{\infty}(x, n)=\kappa_{\infty}(n) x+O\left(n^{\epsilon} x^{\epsilon}\right),
$$

where we need to take $c_{\epsilon} \geq(F(Q)+2)\left(Q^{\epsilon}+1\right) \rho_{\epsilon} /\left(2^{\epsilon}-1\right)^{F(Q)}$. 
The proof of Theorem 14 is complete. The constant implied by the big oh may be taken equal to $(F(Q)+2)\left(Q^{\epsilon}+1\right) \rho_{\epsilon} /\left(2^{\epsilon}-1\right)^{F(Q)}$.

Corollary 14.1. We have

$$
\phi_{\infty}(n, n)=\frac{n^{2} \phi_{\infty}(n)}{\phi_{\infty}\left(n^{2}\right)}+O\left(n^{\eta}\right)
$$

for any $\eta$ with $0<\eta \leq 2$.

Proof. Use Remark 1, Theorem 14, and the fact that $\kappa_{\infty}(n)=n / \sigma_{\infty}(n)$.

Corollary 14.2. We have, where $x>0$,

$$
\psi_{\infty}(x, n) \stackrel{\text { def }}{=} \sum_{\substack{m \leq x \\(m, n)_{\infty}=1}} m=\frac{1}{2} \kappa_{\infty}(n) x^{2}+O\left(x^{1+\epsilon} n^{\epsilon}\right) .
$$

Proof. Use a Stieltjes integration with $\phi_{\infty}(x, n)$ as integrator (maintaining $n$ as a constant parameter).

THEOREM 15. Let $g$ be any bounded arithmetic function and define the arithmetic function $f$ by

$$
f(n)=n \sum_{\left.d\right|_{\infty} n} \frac{g(d)}{d}
$$

Then, for any $x>1$,

$$
\sum_{n \leq x} f(n)=\frac{x^{2}}{2} \sum_{n=1}^{\infty} \frac{g(n) \kappa_{\infty}(n)}{n^{2}}+O\left(x^{1+\epsilon} \log x\right) .
$$

Proof. We have

$$
\begin{aligned}
\sum_{n \leq x} f(n) & =\sum_{n \leq x} n \sum_{\left.d\right|_{\infty} n} \frac{g(d)}{d}=\sum_{n \leq x} \sum_{\substack{d d^{\prime}=n \\
\left(d, d^{\prime}\right)_{\infty}=1}} g(d) d^{\prime} \\
& =\sum_{\substack{d d^{\prime} \leq x \\
\left(d, d^{\prime}\right)_{\infty}=1}} g(d) d^{\prime}=\sum_{d \leq x} g(d) \sum_{\substack{d^{\prime} \leq x / d \\
\left(d^{\prime}, d\right)_{\infty}=1}} d^{\prime} \\
& =\sum_{d \leq x} g(d) \psi_{\infty}\left(\frac{x}{d}, d\right) \\
& =\frac{x^{2}}{2} \sum_{n \leq x} \frac{g(n) \kappa_{\infty}(n)}{n^{2}}+O\left(x^{1+\epsilon} \sum_{n \leq x} \frac{n^{\epsilon}}{n^{1+\epsilon}}\right) \\
& =\frac{x^{2}}{2} \sum_{n \leq x} \frac{g(n) \kappa_{\infty}(n)}{n^{2}}+O\left(x^{1+\epsilon} \log x\right),
\end{aligned}
$$

using Corollary 14.2.

We now have

$$
\sum_{n \leq x} f(n)=\frac{x^{2}}{2} \sum_{n=1}^{\infty} \frac{g(n) \kappa_{\infty}(n)}{n^{2}}+O\left(x^{2} \sum_{n>x} \frac{\kappa_{\infty}(n)}{n^{2}}\right)+O\left(x^{1+\epsilon} \log x\right) .
$$

Since $0<\kappa_{\infty}(n) \leq 1$ and $\sum_{n>x} 1 / n^{2}=O\left(x^{-1}\right)$, the theorem is proved.

Taking, respectively, $g(n)=1$ and $g(n)=\mu_{\infty}(n)$, we obtain the following results. 
Corollaky 15.1. For any $x>1$,

$$
\sum_{n \leq x} \sigma_{\infty}(n)=\frac{x^{2}}{2} \sum_{n=1}^{\infty} \frac{\kappa_{\infty}(n)}{n^{2}}+O\left(x^{1+\epsilon} \log x\right) .
$$

Corollary 15.2. For any $x>1$,

$$
\sum_{n \leq x} \phi_{\infty}(n)=\frac{x^{2}}{2} \sum_{n=1}^{\infty} \frac{\mu_{\infty}(n) \kappa_{\infty}(n)}{n^{2}}+O\left(x^{1+\epsilon} \log x\right) .
$$

We also have

Corollary 15.3. For any $x>1$,

$$
\sum_{n \leq x} n \kappa_{\infty}(n)=\frac{x^{2}}{2} \sum_{n=1}^{\infty} \frac{\mu_{\infty}(n) \kappa_{\infty}^{2}(n)}{n^{2}}+O\left(x^{1+\epsilon} \log x\right) .
$$

Proof. We need first the result

$$
\kappa_{\infty}(n)=\sum_{\left.d\right|_{\infty} n} \mu_{\infty}(d) \frac{\kappa_{\infty}(d)}{d}
$$

This can be proved directly, or by using the Möbius inversion formula (Theorem 9), as follows. Since

$$
\frac{n}{\kappa_{\infty}(n)}=\sigma_{\infty}(n)=\sum_{\left.d\right|_{\infty} n} d
$$

and since $\kappa_{\infty}$ is $I$-multiplicative, we have

$$
n=\sum_{\left.d\right|_{\infty} n} \mu_{\infty}(d) \frac{n / d}{\kappa_{\infty}(n / d)}=\frac{n}{\kappa_{\infty}(n)} \sum_{\left.d\right|_{\infty} n} \frac{\mu_{\infty}(d) \kappa_{\infty}(d)}{d}
$$

giving (3.4).

Now substitute $g(n)=\mu_{\infty}(n) \kappa_{\infty}(n)$ in Theorem 15 to obtain Corollary 15.3.

Set

$$
A=\sum_{n=1}^{\infty} \frac{\kappa_{\infty}(n)}{n^{2}}, \quad B=\sum_{n=1}^{\infty} \frac{\mu_{\infty}(n) \kappa_{\infty}(n)}{n^{2}}, \quad C=\sum_{n=1}^{\infty} \frac{\mu_{\infty}(n) \kappa_{\infty}^{2}(n)}{n^{2}}
$$

These may be approximated using the infinitary analogue of Euler products:

THEOREM 16. If $f(n)$ is an I-multiplicative arithmetic function such that the series $\sum f(n) / n^{s}$ is absolutely convergent, then

$$
\sum_{n=1}^{\infty} \frac{f(n)}{n^{s}}=\prod_{P \in I}\left(1+\frac{f(P)}{P^{s}}\right)
$$

Proof. The proof is analogous to, but easier than, that of Theorem 11.7 in [3].

Using the definitions of $\kappa_{\infty}$ and $\mu_{\infty}$, we thus have

$$
A=\prod_{P \in I}\left(1+\frac{1}{P(P+1)}\right), \quad B=\prod_{P \in I}\left(1-\frac{1}{P(P+1)}\right), \quad C=\prod_{P \in I}\left(1-\frac{1}{(P+1)^{2}}\right) .
$$

In this way, we have calculated the following numerical values for the coefficients in Corollaries $15.1,15.2$ and 15.3 , respectively:

$$
\frac{A}{2} \doteq 0.73, \quad \frac{B}{2} \doteq 0.33, \quad \frac{C}{2} \doteq 0.37
$$

The results of Corollaries 15.1 and 15.2 may now be compared with the following results for ordinary and unitary divisors. The superscript * refers to unitary divisors; see E. Cohen [4] for 
the corresponding definitions and results. See [3] for the results concerning ordinary divisors.

$$
\begin{aligned}
\sum_{n \leq x} \sigma(n) & =\frac{1}{2} \zeta(2) x^{2}+O(x \log x), & \frac{1}{2} \zeta(2) & \doteq 0.82 \\
\sum_{n \leq x} \sigma^{*}(n) & =\frac{\pi^{2}}{12 \zeta(3)} x^{2}+O\left(x \log ^{2} x\right), & \frac{\pi^{2}}{12 \zeta(3)} & \doteq 0.68 \\
\sum_{n \leq x} \phi(n) & =\frac{3}{\pi^{2}} x^{2}+O(x \log x), & \frac{3}{\pi^{2}} & \doteq 0.30 \\
\sum_{n \leq x} \phi^{*}(n) & =\frac{\alpha}{2} x^{2}+O\left(x \log ^{2} x\right), & \frac{\alpha}{2}=\frac{1}{2} \prod_{p \text { prime }}\left(1-\frac{1}{p(p+1)}\right) & \doteq 0.35
\end{aligned}
$$

We now proceed to the evaluation of $\sum_{n \leq x} \tau_{\infty}(n)$.

LEMMA 2. With $C$ as above, we have, for all $x>1$,

$$
\begin{gathered}
\sum_{n \leq x} \kappa_{\infty}(n)=C x+O\left(x^{\epsilon} \log x\right) \\
\sum_{n \leq x} \frac{\kappa_{\infty}(n)}{n}=C \log x+C^{\prime}+O\left(x^{-1+\epsilon} \log x\right),
\end{gathered}
$$

where $C^{\prime}$ is a constant to be described.

Proof. We use as an integrator the function $K$ defined by

$$
K(x)=0 \text { if } x \leq 1, \quad K(x)=\sum_{n \leq x} n \kappa_{\infty}(n)=\frac{C}{2} x^{2}+O\left(x^{1+\epsilon} \log x\right) \text { if } x>1 .
$$

(See Corollary 15.3.)

(i)

$$
\begin{aligned}
\sum_{n \leq x} \kappa_{\infty}(n) & =\int_{1}^{x} \frac{d K(u)}{u}=\frac{K(x)}{x}+\int_{1}^{x} \frac{K(u)}{u^{2}} d u \\
& =\frac{C}{2} x+O\left(x^{\epsilon} \log x\right)+\int_{1}^{x}\left(\frac{C}{2}+O\left(u^{-1+\epsilon} \log u\right)\right) d u \\
& =C x+O\left(x^{\epsilon} \log x\right) .
\end{aligned}
$$

(ii)

$$
\begin{aligned}
\sum_{n \leq x} \frac{\kappa_{\infty}(n)}{n}= & \int_{1}^{x} \frac{d K(u)}{u^{2}}=\frac{K(x)}{x^{2}}+2 \int_{1}^{x} \frac{K(u)}{u^{3}} d u \\
= & \frac{C}{2}+O\left(x^{-1+\epsilon} \log x\right)+2 \int_{1}^{x}\left(\frac{C}{2 u}+\left(\frac{K(u)}{u^{3}}-\frac{C}{2 u}\right)\right) d u \\
= & \frac{C}{2}+O\left(x^{-1+\epsilon} \log x\right)+C \log x+\int_{1}^{\infty} 2\left(\frac{K(u)}{u^{3}}-\frac{C}{2 u}\right) d u \\
& -2 \int_{x}^{\infty}\left(\frac{K(u)}{u^{3}}-\frac{C}{2 u}\right) d u
\end{aligned}
$$

Since $K(x) / x^{3}-C / 2 x=O\left(x^{-2+\epsilon} \log x\right)$, the first integral here is convergent and hence is a constant (namely, $\left.C^{\prime}-\frac{1}{2} C\right)$, and the second integral is $O\left(\int_{x}^{\infty} u^{-2+\epsilon} \log u d u\right)=O\left(x^{-1+\epsilon} \log x\right)$.
The result follows. 
THEOREM 17. We have, for any $x>1$,

$$
\sum_{n \leq x} \tau_{\infty}(n)=C x \log x+\left(2 C^{\prime}-C\right) x+O\left(x^{\frac{1}{2}+\epsilon}\right)
$$

Proof. Having developed Theorem 14 and Lemma 2, the proof follows standard lines:

$$
\begin{aligned}
\sum_{n \leq x} \tau_{\infty}(n)= & \sum_{n \leq x} \sum_{\left.d\right|_{\infty} n} 1=\sum_{n \leq x} \sum_{\substack{d d^{\prime}=n \\
\left(d, d^{\prime}\right)_{\infty}=1}} 1=\sum_{\substack{d d^{\prime} \leq x \\
\left(d, d^{\prime}\right)_{\infty}=1}} 1 \\
= & 2 \sum_{\substack{d d^{\prime} \leq x, d \leq \sqrt{x} \\
\left(d, d^{\prime}\right)_{\infty}=1}} 1-\sum_{\substack{d \leq \sqrt{x}, d^{\prime} \leq \sqrt{x} \\
\left(d, d^{\prime}\right)_{\infty}=1}} 1 \\
= & 2 \sum_{d \leq \sqrt{x}} \sum_{\substack{d^{\prime} \leq x / d \\
\left(d^{\prime}, d_{\infty}=1\right.}} 1-\sum_{d \leq \sqrt{x}} \sum_{\substack{d^{\prime} \leq \sqrt{x} \\
\left(d^{\prime}, d\right)_{\infty}=1}} 1 \\
= & 2 \sum_{n \leq \sqrt{x}} \phi_{\infty}\left(\frac{x}{n}, n\right)-\sum_{n \leq \sqrt{x}} \phi_{\infty}(\sqrt{x}, n) \\
= & 2 \sum_{n \leq \sqrt{x}}\left(\kappa_{\infty}(n) \frac{x}{n}+O\left(n^{\epsilon}\left(\frac{x}{n}\right)^{\epsilon}\right)\right)-\sum_{n \leq \sqrt{x}}\left(\kappa_{\infty}(n) \sqrt{x}+O\left(n^{\epsilon} x^{\frac{1}{2} \epsilon}\right)\right) \\
= & 2 x \sum_{n \leq \sqrt{x}} \frac{\kappa \infty(n)}{n}+O\left(x^{\frac{1}{2}+\epsilon}\right)-\sqrt{x} \sum_{n \leq \sqrt{x}} \kappa_{\infty}(n)+O\left(x^{\frac{1}{2} \epsilon} \sum_{n \leq \sqrt{x}} n^{\epsilon}\right) \\
= & 2 x\left(C \log \sqrt{x}+C^{\prime}+O\left(x^{\frac{1}{2}(-1+\epsilon)} \log x\right)\right)+O\left(x^{\frac{1}{2}+\epsilon}\right) \\
& \quad-\sqrt{x}\left(C \sqrt{x}+O\left(x^{\frac{1}{2} \epsilon} \log x\right)\right)+O\left(x^{\frac{1}{2} \epsilon} x^{\frac{1}{2}(1+\epsilon)}\right) \\
= & C x \log x+\left(2 C^{\prime}-C\right) x+O\left(x^{\frac{1}{2}+\epsilon}\right) .
\end{aligned}
$$

For ordinary divisors, the result corresponding to that in Theorem 17, as originally obtained by Dirichlet, is

$$
\sum_{n \leq x} \tau(n)=x \log x+(2 \gamma-1) x+O\left(x^{\frac{1}{2}}\right),
$$

where $\gamma$ is Euler's constant. (It is well known that the error term has subsequently been improved. This is the classical divisor problem, discussed in [3].) For unitary divisors, we have the following result (obtained by Gioia and Vaidya [5]):

$$
\sum_{n \leq x} \tau^{*}(n)=\frac{x}{\zeta(2)}\left(\log x+2 \gamma-1-\frac{2 \zeta^{\prime}(2)}{\zeta(2)}\right)+O\left(x^{\frac{1}{2}}\right) .
$$

\section{REFERENCES}

1. COHEN, G. L. On an integer's infinitary divisors, Math. Comp. 54 (1990) 395-411.

2. HAGIS, P., Jr., and COHEN, G. L. Infinitary harmonic numbers' Bull. Austral. Math. Soc. 41 (1990) 151-158.

3. APOSTOL, T. M. Introduction to Analytic Number Theory, Springer-Verlag, New York, 1976.

4. COHEN, E. Arithmetical functions associated with the unitary divisors of an integer, Math. Zeit. 74 (1960) 66-80.

5. GIOIA, A. A. and VAIDYA, A. M. The number of squarefree divisors of an integer, Duke Math. J. 33 (1966) 797-799. 


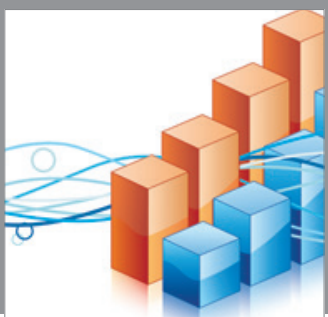

Advances in

Operations Research

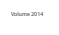

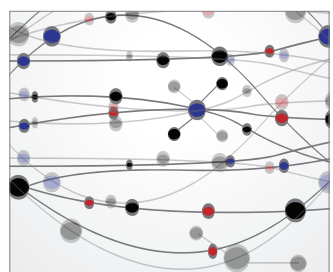

\section{The Scientific} World Journal
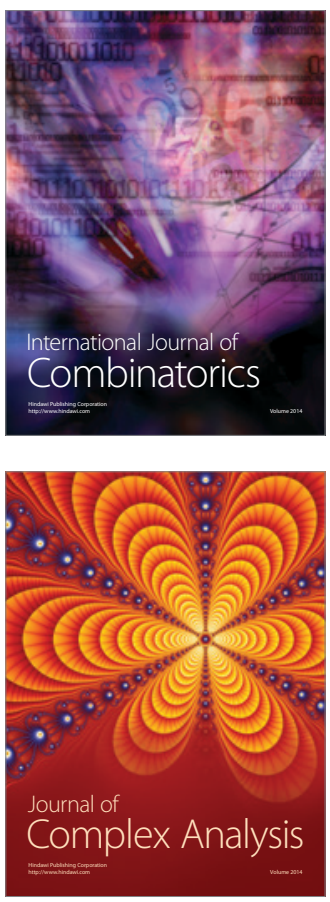

International Journal of

Mathematics and

Mathematical

Sciences
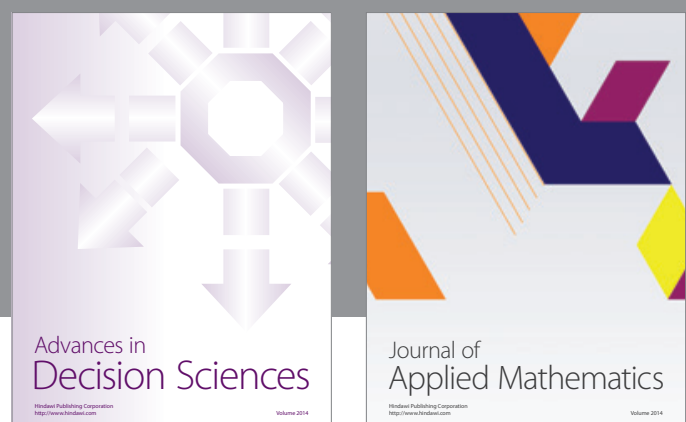

Journal of

Applied Mathematics
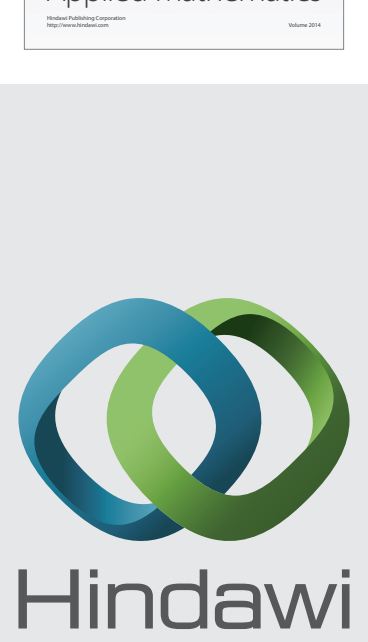

Submit your manuscripts at http://www.hindawi.com
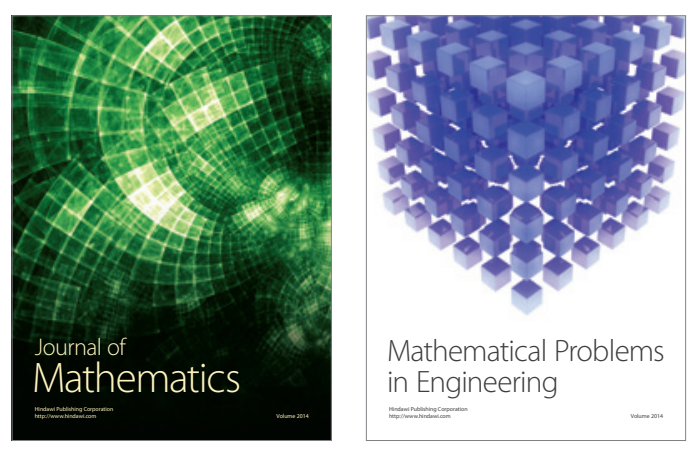

Mathematical Problems in Engineering
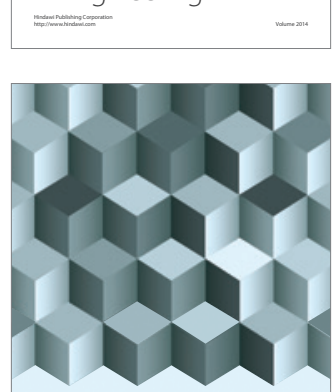

Journal of

Function Spaces
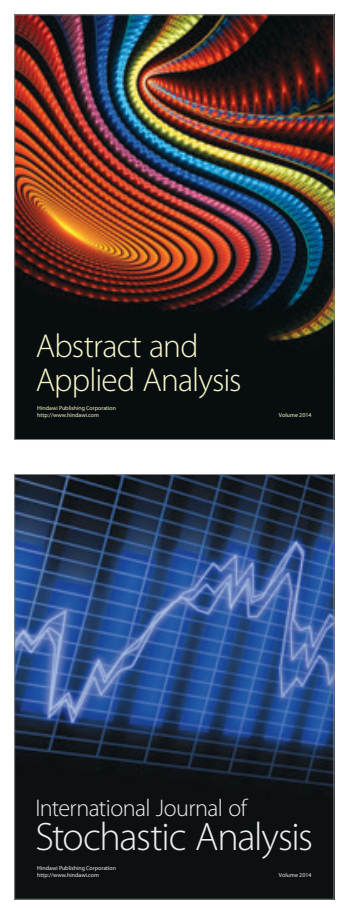

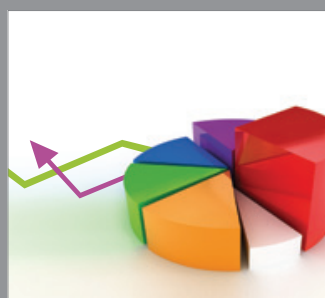

ournal of

Probability and Statistics

Promensencen
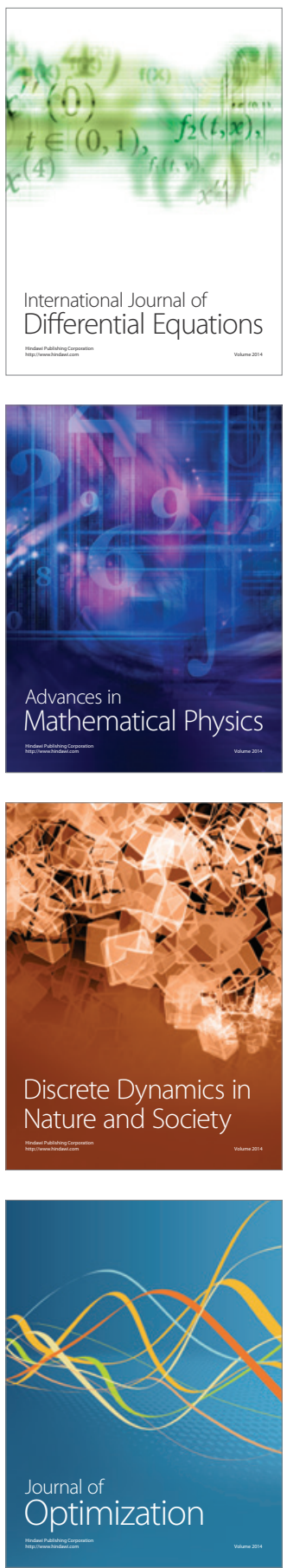